\title{
Tintoretto.
}

\section{Kritische Studien über des Meisters Werke}

von Henry Thode.

Keiner unter den grossen Meistern der italienischen Renaissance ist so lange von der kunstgeschichtlichen Forschung unberücksichtigt geblieben, als der Künstler, in dessen gewaltigen Schöpfungen doch alle Bestrebungen der venezianischen Malersehule gipfeln: Tintoretto. Ein seit Vasari's Zeiten mit nur geringen Modificationen sich fortpflanzendes Vorurtheil, welches den unvergleichlich kühnen Schöpfer überwältigend eindrucksvoller Werke zu einer Erscheinung der beginnenden „Verfallszeit" stempelte und mit unbegreiflicher Kritiklosigkeit seine Bedeutung nur im Decorativen suchte, hat von einer gründlichen Beschäftigung mit seinen Werken abgeschreckt. Nur Wenige sind dem Geheimniss dieser Kunst nahegetreten, und selbst John Ruskin, dem wir die ersten Hinweise auf dasselbe verdanken, verfolgte den von ihm eingeschlagenen Weg nicht, sondern übertrug, immer wieder in das Aeusserliche sich verirrend, die Widersprüche seines geistreichen, aber sehr subjectiv willkürlichen Empfindens auf das Schaffen eines Meisters, der, wenn Einer, seine Kunst zu einem einheitlichen Ausdruck seiner Ideen gemacht hat. Statt jener Anregung Ruskin's folgend nun als die wichtigate Aufgabe eine gewissenhafte historisehe und analytische Untersuchung über die Werke Robusti's anzustreben, haben sich die zwei englisehen Schriftsteller, welche kurze Biographien desselben schrieben: W. Roscoe Osler (in ,The great artists", London 1879) und Frank Preston Stearns (New-York 1894) wiederum daran genügen lassen, bloss die allgemein bekannten Gemälde zu beschreiben und Betrachtungen aesthetischer Art anzustellen, welche wohl manches Gute enthelten, das Verständniss des grossen Problemes, um das es sich hier handelt, aber nicht wesentlich förderten. Ein Fortschritt gegenüber der früheren Biographie aus Eubert Janitsehek's Feder (in „Kunst und Künstler" 1879), welche sich gleichfalls nur auf wenige Hauptwerke beschrönkt, machte sich bei ihnen nur in dem Versuche einer gerechteren Würdigung des Meisters geltend.

So blieb denn alle eigentliche Arbeit noch zu leisten. Zahlreiche, 
zum Theil höchst bedeutende, bisher von den Besuchern der Kirchen und Galerien kaum oder gar nicht beachtete Gemälde waren im eigentlichen Sinne des Wortes zu entdecken, der Ueberblick iiber die gesammte, fast übermenschliche Thätigkeit Robusti's war zu gewinnen, die Entwickelung seines Stiles, die bei dem Mangel an Daten freilich nur durch immer erneuten kritischen Vergleich unter bedeutenden Schwierigkeiten erkannt werden konnte, musste dargelegt, die Sonderung seiner Werke von denen des Sohnes und seiner anderen Schüler vorgenommen, bei vielen stark übermalten Bildern eine geistige Reconstruction ihres einstigen originalen Anssehens versucht werden, und endlich geit es, die Ideenwelt, die sich in diesen Werken spiegelte, zu ergriinden und enthïllen.

Die Resultate solcher langjährigen Arbeit habe ich in meiner soeben erscheinenden Biographie Tintoretto's (in den Künstlermonographien Velhagen und Klasing's) zusammengefasst und der Oeffentlichkeit übergeben. Dass es sich bei ihr nur um die Studie zu einem grösseren Werk handle, dass nur die wichtigsten Fragen ausgearbeitet werden konnten, vieles Besondere aber, sowohl was die einzelnen Gemälde als auch was kritische Untersuchungen anbetrifft, bei Seite gelassen oder doch stark condensirt wurde, muss auch hier wieder hervorgehoben werden. Die Bewältigung einer so grossen Fülle zumeist unbearbeiteten Stoffes musste auf einmal und in so eng gegebenen Grenzen unmöglich sein. Doch darf ich wohl glauben, das Bild des Meisters und seines Schaffens in den charakteristischen und wesentlichen Umrisslinien gestaltet und überzeugend nachgewiesen zu haben, wie ganz seine Kunst von der immer wieder an ihm getadelten Willkür frei gewesen ist, wie ganz ihre Sprache in Form, Farbe und Licht nur der adäquate Ausdruck der ihm eigenthümlichen erhabenen Ideen war und wie wir in ihm den letzten, höchsten, zusammenfassenden Ausdruck aller künstlerischen Bestrebungen der Renaissance zu erkennen haben. Statt des frivol die Souverainetät seines Könuens missbrauchenden Virtuosen tritt uns einer der tiefsinnigsten Künstler entgegen, welcher, um eine Natur und Geist, Philosophie und Religion harmonisch umschliessende freieste Weltanschaung zu verdeutlichen, auch aller zur höchsten Freiheit und Universalität enwickelten Ausdrucksmittel der Kunst sich mit absoluter Herrscherkraft bedienen musste.

Wohl weiss ich, dass einer solohen neuen Auffassung nicht überall, nicht gleich Verständniss begegnen wird - die Macht dreihundertjähriger Tradition steht ihr entgegen. Bei Manchen aber, am ersten zunz̈chst wohl bei Künstlern, wird sie denn doch ein williges Entgegenkommen finden. Und wer sich mit dem Verfasser wirklich in die Betrachtung jener Wunderwerke versenkt, wird mit ihm das Bekenntuiss, das sie von ihrem Meister ablegen, in ergreifenden Augenblicken der Entruckung empfangen.

Den kunsthistorischen Forschern aber soll im Folgenden die Begründung der in der Monographie aufgestellten Behauptungen gegeben werden, indem ein erstes kritisches Verzeichniss der Werke Tintoretto's aufzustellen versucht wird. Dasselbe ist noch mancher Ergänzung bedürftig. 
Mancherlei Aufklïrungen, hoffentlich auch wichtige neue Lebensthatsachen, werden wir aller Voraugsicht nach dem unermüdliehen Spender aus den Archivschätzen der Frari, Pietro Paoletti, und seinem Bundesgenossen Gusta Ludwig dereinst gu verdanken haben.

I.

Chronologisches.

Die älteste Quelle, der wir Nachrichten von Tintoretto entnebmen, sind die zwei an ihn gerichteten Briefe Pietro Aretino's aus den Jahren 1545. (Februar) und 1548 (April). (Pariser Ausgabe der lettere IV, S. 110, Bottari III, 126 und IV, 181, Bottari III, 161). Der erstere bespricht die für Aretino ausgeführten Bilder: "A pollo und Marsyas" und "Mercur und Argo", der andere "die Befreiung des Sklaven durch den heiligen Marcus." Die nächste Nachricht finden wir in Anselmo Guisconi's Dialogo: Tutte le cose notabili e belle che sono in Venezia von 1556 (herausgegeben nach einem Exemplar der ersten Ausgabe in der Bibliothek des Museo Correr von Andrea Battaggia 1861, Tipografia Emiliana). Hier heisst es (S. 13): nè vi voglio lasciare a dietro Jacomo Tintoretto, il quale è tutto spirito e tutto prontezza. Questi ha un suo quadro in consiglio, et ha diverse opere per tutta la città, ma si desidera in lui più diligenza, che del resto è eccellente. - F. Voi dite il vero; anch io ho considerato il suo quadro, non pare finito; per ciò credo che questo nasca dalla sua molta prestezza. - V. Cos̀ è." Das Bild im grossen Rathssaal muss die später verbrannte „Krönung Barbarossa's" gewesen sein. - In Francesco Sansovino's "Cose notabili che sono in Venetia" 1581, welche an Guisconi anknüpfen, findet sich nichts Neues. - Es folgen die Nachrichten in Vasari's Vite zweiter Auflage 1568. Hier werden folgende. Werke Jacopo's erwähnt: die Krönung Barbarossa's und die Excommunication desselben in der Sala del gran Consiglio, zwei Legenden des h. Rochus (die eine: Rochus im Hospital), und die Heilung des Gichtbrïchigen in S. Rocco, Moses empfängt die Gesetzestafeln, das jüngste Gericht, der Tempelgang Mariens und die Bekehrung Saul's (an den Orgelflügeln) in der Madonna dell' Orto, die Kreuzabnabme in der Carità, Moses in der Wüste in der Sacristei von S. Sebastiano; in S. Giobbe am Altar der Pietà: die drei Marien und die Heiligen Franz, Sebastian und Johannes der Täufer; in den Servi an den Orgelflügeln: die Heiligen Augustin und Filippo, sowie Kain's Brndermord; in S. Felice an der Decke der Tribuna: die vier Evangelisten, in einer Lunette: die Verkündigung, in einer anderen: das Gebet in Gethsemane, an der Eingangswand: das Abendmahl; in S. Francesco della Vigna: die Kreazabnahme; die vier Legenden des $\mathrm{h}$. Marcus in der Scuola di San Marco, und ebendaselbst das Altarbild eines $h$. Marcus. In der Scuola di $\mathbf{S}$. Rocco: die Kreuzigung und die Deckenbilder im gleichen Raume. - Alle diese Werke sind also vor 1588, resp. 1566 Mai (Vasari in Venedig) entstanden.

In der Vevetia descritta des Sansovino 1581 werden dann eine 
gröвsere Anzahl anderer Werke verzeichnet, ohne dass uns aber im Einzelnen bemerkenswerthe Daten gegeben würden.

In Jahre 1587 veröffentlichte Girolamo Bardi Fiorentino, welcher an der Auswahl und Bestimmung der Darstellungen als Berather neben Jacopo Contarini und Jacopo Marcello selbst thätig gewesen war, seine „Dichiaratione di tutte le istorie che si contengono nelle sale dello Scrutinio et del gran Consiglio del Palagio Ducale." Seine Angaben über die Künstler sind nur mit grosser Vorsicht aufzunehmen; er hat sich offenbar an den ersten Plan der Vertheilung der Aufgaben an die verschiedenen Naler gehalten, welcher dann später nicht festgehalten worden ist. Fr nennt als Werke Tintoretto's: die Schlscht bei Zara, den Sieg über die Türken bei Curzolari und das Jüngste Gericht über dem Tribunal, alle drei in der Sala dello Scrutinio; im grossen Rathssaal, Wandbilder: die Ankunft der Gesandten in Pavia, Alessio's Besuch der Kreuzfahrer in Zara, Alessio's Tod and die zweite Einnahme von Constantinopel; Deckenbilder: die Vertheidigung Brescia's, die Seeschlacht auf dem Gardasee, der Sieg Vittorio Soranzo's izber den Herzog von Ferrara und Jacopo Marcello's Sieg über die Arragonesen, endlich das grosse Deckengemälde mit der Allegorie Venezia's; ausserdem alle die Dogenbildnisse am Fries in beiden Sälen. Von diesen Bildern werden von Ridolfi und Martinioni nur die Schlacht bei Zara, die Ankunft der Gesandten in Pavia und die Deckenbilder Jacopo zugeschrieben: die Schlacht bei Curzolari ist von Andrea Vicentino, das Jüngste Gericht von Palma giovane, Alessio in Zara von Andrea Vicentino, Alessio's Tod und die Einnahme von Constantinopel von Domenico Tintoretto. Von den Dogenbildnissen am Friese ist nach den beiden Schriftstellern nur ein Theil von Jacopo gemalt worden.

$\mathrm{Zu}$ diesen zeitgenössischen Mittheilungen gesellen sich nun weiter einige Daten, die man Documenten (die „Ricevute“ und Zahlungsanweisungen an Tintoretto für die Arbeiten in S. Roceo sind in der Scuola susgestellt), Bilderaufschriften und sonstigen Notizen entnehmen kann. Ziehen wir dieselben mit in Betracht, so ergiebt sich folgende chronologische Liste:

1518. Jacopo Robusti, Sohn des Färbers Battista Robusti, geboren. - Ridolf's Angabe 1512 ist als irrig von Galanti (Discorso: il Tintoretto, atti della R. Accademia di Belle Arti, Venedig 1876) erwiesen worden, welcher aus dem Necrologio dei Proveditori alla Sanità die Notiz publizirte: adi majo 1594. El Magnifico messer Jacomo di Robusti ditto el Tentoretto de anni 75 da febre giorni 15, $\mathrm{S}$. Marcilian. - Eine übereinstimmende Angabe hatte schon Giov. Prosdocimo Zabeo in seinem Elogio a Giacomo Robusti, Venezia 1814 aus den Acten der Sacristei von S. Marziliano veröffentlicht: 31 majo 1594 . Morto mes. Jacomo Robusti ditto Tentoretto de età de anni 75 e m. 8 ammalato giorni quindese da frieve. S. Marcellian. - Demnach wäre Tintoretto also Ende September oder Anfang November 1518 geboren worden.

1535 circa - 1545. Lernzeit und erste Thätigkeit. 
1545. Zwei Gemälde für Pietro Aretino: Apollo und Marsyas und Merkur und Argo. (Lettere, Pariser Ausgabe IV. S. 110. Bottari III, 126.) 1547. Das Abendmahl in S. Marcuola. Nach der Bezeichnung: 1547 die 17 Agosto in tempo de miser Isepo Morandelo et compagni.

1547. Porträt eines venezianisehen Nobile in Besitze des Baron von Liphart in Rathshof bei Dorpat. Vergl. W. Neumann: Aus baltisahen. Galerien in der Zeitschrift für bildende Kunst 1900, 8. 271. Mit dieser Jahreszahl bezeichnet.

1547. Portrait des Tommaso Mocenigo. In der Akademie zu Wien.

1548. Die Befreiung des Sklaven durch den h. Marcus, für die Scuola di S. Mareo. Jetzt in der Akademie zu Venedig. (Pietro Aretino's Brief. Bottari III, 161.)

1548. Männliches Porträt bei Mr. Holford in London. Mit der Jahreszahl bezeichnet.

1551. Porträt des Pietro Aretino. Erwähnt in einem Briefe des Francesco Marcolino an diesen vom 15. September 1551. (Bottari I, 523).

1553. Männliches Bildniss. In der k. k. Galerie zu Wien, Nr. 250.

Zwischen 1545 und 1553. Porträt des Dogen Francesco Donato, das er gemalt hat und das vermuthlich während der Regierungszeit Donato's entstanden sein wird.

Vor 1556. Die Krönung Barbarossa's in der Sala del gran Con siglio, 1577 verbrannt. Nach Guisconi.

1555. Männliches Bildniss in der Galerie zu Dublin.

1557. Porträt des Tommaso Contarini im Dogenpalast.

1558? 1559? Verheirathet er sich mit Faugtina Vescovi.

1559. Ein Bild für S. Rocco „dove si mette li arzenti presso il Christo". Die Heilung des Gichtbrüchigen. In einer anderen Notiz wird das Bild il quadro di arzentari genannt. (Nach Zahlungsanweisungen vom 2. April und 15. October in der Scuola di S. Rocco ausgestellt.) S. auch Nicoletti : Illustrazione della Chiesa und Scuola di S. Rocco 1885, S. 60.

1560. Seine Tochter Marietta geboren.

1560. Bildniss des Dogen Girolamo Priuli. Er erhält dafür am 23. Dezember 25 Ducaten (Lorenzi: Monumenti per servire slla storia del Palazzo duesle 8. 307). Ein Bildniss des Priuli befindet sich in der k. k. Galerie zu Wien.

1560. Porträt des Ottaviano Grimani. Wien, Akademie. Mit Jahreszahl bezeichnet.

1560? Um 1560 nach Ridolfi angeblich die Deckenmalereien im Sitzungasaale der Scuola di 8. Roceo. Nach Vasari wären dieselben nach der Kreuzigung 1565 entstanden. Nach einem Zahlungasus weis hat in der That Jacopo die Bilder der Schule geschenkt (Nicoletti S. 59).

1561. Die Hochzeit von Kana. In S. Maria della Salute.

1561. Thätigkeit in der Libreria von S. Marco, mit Paolo Veronese, Schisvone, Zelotti u. A.

1562. 21. Juni erhält der Guardian grando der Scuola di S. Mareo 
die Erlaubniss, auf seine Kosten für den grossen Sasl drei Gemälde mit den Wundern des $h$. Marcus (auch die sieben Tugenden und die sieben Laster, sowie den triumphirenden h. Marcns) ausftihren zu lassen. Es sind die drei Legenden (Palazzo resle und Brera), die Tintoretto gemalt hat, und zwar demnach wohl 1562 und in den folgenden Jahren. (Galanti S. 85.)

1562. Sein Sohn Domenico geboren.

1568. Jacopo sendet an den Cardinal Ercole Gonzaga am 9. Mai eine von diesem 1581 bestellte kleine "Türkenschlacht" und schreibt dazu: desidereri bene che Vostra Illustrissima Signoria $m$ ' adoperasse in cosa che fosse de mia maggiore professione che di fare queste figure piccole. Però accetterà il buon animo; et se son stato in servirla, ne fu cagione la difficult dell' opera, la quale ricerea lunghezza di tempo. (A. Luzio in Archivio storico dell' arte III. S. 207).

1562. Am 7. Januar wird beschlossen, die drei fehlenden Wandgemälde im grossen Rathssaale ausführen zu lassen (Lorenzi, Monumenti S. 300 f.) Eines derselben: „Die Excommunication, Barbarossa's“, 1577 verbrannt, ward von Tintoretto gemalt, 1562, denn am 23 . October wurde den. Malern eine Zahiung gemacht. (Lorenzi S. 312.)

1562? 1563? Das Deckenbild: „Girolamo Prinli erhält das Sehwert von der Gereehtigkeit", in dem Salotto d'ingresso. Dieser Raum wird wohl 1562 und 1583 fertig gestellt. (Lorenzi 8. 311, 314.)

1563. Ist Jacopo mit Tizian und Anderen Schiedsrichter über die Mosaiken der Zuceati in S. Marco. (Zanetti, Pitt. Ven. S. 725. Protokoll bei Harzen: Schiavona im deutschen Kunstblatt 1853.)

1563. Ist er Schiedsrichter mit Paolo Veronese und Sansovino über die Concurrenzarbeiten in Mosaik von Francesco Zuccato, Giannantonio Bianchini, Bartolommeo Bozza und Domenico Bianchini, (Stringa: Aggiunte al Sansovino. Venezia 1604, S. 69 und Saceardo: les Mosaiques de Saint Marc. 1896. S. 89, 90.)

1565. Altargemälde in San Casciano: „Der auferstehende Christus mit dem Heiligen Cassian und Cäcilia". Das Bild trug früher eine Bezeichnung. (Moschini: Guida di Venezia II, 129).

1565. Die Kreuzigung in der Senola di S. Roceo. Bezahlt mit 250 Dukaten am 9. Mai 1566. Bezeichnet.

1565. Männliches Bildniss in der Galerie zu Darmstadt, mit der Jahreszahl bezeichnet (gen. Tizian).

1506. „Die Madonna und die drei Tesorieri aus dem Magistrato dei Camerlenghi. Jetzt in der Akademie zu Venedig N. 210. Bezeichnet mit der Jahreszahl.

1566? Wohl bald nach 1565. Christus vor Pilatus und Kreuztragung im Sitzungssaal der Scuola di S. Rocco. (Bezahlung von $134 \mathrm{Du}-$ katen. Nicoletti. S. 58.)

1566. Igt Jacopo Sindico in der Scuola di S. Raceo. (Zabeo S. 52.) 1567. Drei Bilder für die Kirche und Scuola di $\mathrm{S}$. Rocco. Im 
Chor: Rochus im Hospital und seine Gefangennahme. Das dritte war ein Tympanon uiber dem Portal der Scuola, das apäter beim Nenban des Portals entfernt wurde. (Zabeo S. 52. Nicoletti, Illustraxione di S. Rocco. 8. 59.)

1567. Bildnis des Ottavio da Strada. Bei Mr. Salting in London. 1567. Beginn der Malereien an der Decke des oberen Saales in der Seuola di S. Roceo (nach Zabeo.)

1568. Fertigt er die Kartons für zwei Mosaiken (1. Querschiff) in S. Marco: das Abendmahl (auggefuhrt von Domenico Bianchini) und die Hochzeit in Kana (ausgeführt von Bartolommeo Bozzr.) (Saccardo: les mosaiques S. 64, 298.)

1588. Die Kreuzigung und Christi Höllenfahrt in San Cassiano. (Mosehini: Guida di Venezia II, 130.)

In die Zeit bis 1568 fallen nun, da gie von Vasari erwähnt werden, folgende Bilder:

Die beiden grossen Wandgemälde: das goldene Kalb und das Jüngste Gericht in der Madonna dell' Orto.

Der Tempelgang Maria's, ebendaselbst.

Die Bekehrung Sanl's, ebendaselbst. Hier begeht Vasari wohl einen Irrthum. In der Madonna dell' Orto befand sich kein solches Bild, wohl aber in S. Maria Zobenigo an den Orgelflügeln.

Die Kreuzabnshme in der Carità.

Die Orgelflügel in den Servi: Heilige Augustin und Filippo, sowie Kain's Brudermord (versehollen.)

Die vier Evangelisten in S. Felice. Wird von späteren Schriftstellern nicht mehr erwähnt.

Die Verkündigung, ebendaselbst.

Das Gebet in Gethremane, ebendaselbst.

Das Abendmahl, ebendaselbst.

Alle diese Bilder sind heute nicht mehr in S. Felice.

Die Kreuzabnahme in S. Francesco della Vigna.

Moses und die eherne Schlange in der Sacristei von S. Sebagtiano.

Das von Vasari genannte Bild in S. Giobbe hat sich dort nie befunden. Hier liegt offenbar ein Irrthum Vasari's vor.

1567-1577. Deckenmalereien in dem oberen Sasle der Scuola di S. Rocco.

1567-1570. Bildniss des Dogen Pietro Loredano, dessen Regierungszeit in diese Jahre fällt.

1570. Mosaik des Arminio Zuccato: das Paradies in S. Pietro di Castello, angeblich nach Zeichnung ron Jacopo.

1570. Portrait des Lorenzo Amulio im Dogenpalast.

1570-1577. Portrait des Dogen Alvise Mocenigo. In der Akademie zu Venedig.

1571. Die Gewaltthat Tarquin's (Madrid) an Philipp II gesandt. (Carducho dialogos p. 349.) 
1571. 8. November. Beschluss der Signoria, ein grosses Gemälde der Schlacht von Lepanto für die Sala dello Serutinio malen zu lassen. (Lorenzi: Monumenti S. 872.)

1571. Wohl damals die Portraits des Sebastian Veniero, Sieger von Lepanto, als Feldherrn gemalt.

1573. Portrait des Andrea Dolfino im Dogenpalast.

1573. 9. März wird die ,Schlacht bei Lepanto" als fertig erwähnt, und wurde beschlossen, die Mauer, auf welche das Gemälde gehängt werden soll, mit Holz zu verkleiden. (Lorenzi \$. 375.)

1574. 27. September. Tintoretto hat zwei Schreiben an dio Signoria gesandt, in welchen er erwähnt, dass er "die Schlacht von Lepanto “ der Signoria zum Geschenk gemacht hat, seine Dienste fernerhin ohne ein Honorar, (sondern nur die Kosten von Leinwand und Farben) zu verlangen, anbietet und die nächst frei werdende Sensaria im Fondaco dei Tedegchi erbittet. Es wird beschlossen, ihm die Anwartschaft auf eine solche zu gewähren. (Lorenzi \$. 391.) Das Gemälde ist 1577 verbrannt.

1574. Ende Juli. Portrait des Königs Henri III. Der König traf am 17. Juli ein und blieb 11 Tage. (Lorenzi: Mon. S. 396.)

1574. 6. Februar. Contract mit Jeronimo da Mula, welcher bei Jacopo zwei Bilder (jedes mit 20 Figuren) für das grosse Zimmer in seinem Palazzo bestellt: Moses empfängt die Gesetzestafeln und die Auferstehung Lazari. Ausserdem 7 retrati für den Platz über den Thüren. Die Bilder sollen bis zum 15. April fertig sein; der Preis ist 70 Ducaten. (Galanti 8. 78. LiArte II, 500.)

1574? Jedenfalls in den auf 1574 folgenden Jahren malte Tintoretto die Deckenbilder in dem Saale delle quattro porte im Dogenpalast, nach dem. Programm von Francesco Sansovino. 1574 hatte ein. Brand den Raum verheert. (Sansovino, Venezia S. 323.)

1576. Kartons für einzelne der Mosaikdarstellungen aus der Geschichte der Susanna in S. Marco, die von Lorenzo Ceccato ausgeführt wurden. Weitere Zahlungen für diese Arbeit 1587. 1588. (Saceardo: les mosaïques S. 71. 295.)

1576. „Die drei Avogadori vor dem auferstehenden Christus" im Dogenpalast. Mit der Jahreszahl bezeichnetes Bild.

1576. Bildniss des Marco Grimani in der Akademie. Mit der Jahreszahl bezeichnet.

Zwischen 1568 und 1576: Das Jüngste Gericht in der Sala dello Scrutinio im Dogenpalast, das 1577 verbrannte.

1577. Die Versuchung des h. Antonius in S. Trovaso. Wohl in diesem Jahre entstanden, da Antonio Milledonne in diesem Jahre den betreffenden Altar und seine Grabstätte vor demselben stiftete. (Nach der in der Kapelle befindlichen Inschrift.)

1577. Zeichnung für das Mosaik des Paradieses über der Vorhalle von S. Marco, das von Bartolommeo Bozza ausgeführt wurde. Weitere Zeichnungen dafür liefert Jacopo 1588 und 1589. (Saccardo: les mosaïques S. 65. 296.) 
1577. Zeichnung für einen Engel, der von Giannantonio Marini in Mosaik unterhalb der Hochzeit von Kana ausgefuhrt wird, in S. Marco. (Saccardo S. 68. 296.)

1577. Die Portraits der drei Procuratoren Soranzo, Barbaro und Dolfin werden ihm in Auftrag gegeben. (Saccardo S. 296.)

1577. 27. November reicht Jacopo eine Bittschrift an die Confraternità der Scuola di San Rocco ein. Er verspricht, auf seine Kosten die Deckengemälde im oberen Sasle zu vollenden, zehn Wandgemälde und das Altarbild zu malen, sowie die Bilder, die für die Kirche gewünscht werden sollten, auch verpflichtet er sich, jährlich zum Rochusfeste drei grosse Bilder fertig zu liefern, falls ihm für seine Lebensdauer eine jährliche Provision von 100 Dueaten gewährt würde. Am 2. December wird ihm dieselbe zugesichert. (Zabeo. - Giambat. Soravia: le chiese di Venezia III, 105. - Nicoletti: IIl. di S. Roceo S. 60.) Am 3. December erhält er die ersten 100 Ducaten.

1577 und folgende Zeit: Vollendung der Decke im oberen Saale der Scuola, die Ausführung der Gemälde: der h. Rochus und die Thiere, der Tod des h. Rochus, die Verkündigung und Rochus vor dem Papste in der Kirche S. Roceo.

1577. 1578. Das Portrait des Dogen Sebastiano Venier.

1578. Entstehen die vier mythologischen Darstellungen für den Salotto dorato im Dogenpalast: Ariadne und Bacchus, die drei Grazien, Schmiede Vulcan's, Athena vertreibt Mars. Am 26. Juli geben Paolo Veronese und Giacomo Palma ihr Gutachten ab, und am 10. November wird bestimmt, dass Tintoretto für jedes 50 Dueaten erhalte. (Lorenzi: Momumenti S. 449.)

1578? 1579? Tintoretto malt für Guglielmo Gonzaga, Herzog von Mantua, Bilder. Sie werden ohne nähere Angabe in einem Briefe des Conte Sangiorgio an Mons. Paolo Moro (in Venedig), welcher weitere Gemälde bestellt, am 1. October 1579 genannt. (Al. Luzio: fasti Gonzagheschi dipinti dal Tintoretto, im Archivio storico dell' arte III, 397.) Nach Luzio's glaubwürdiger Vermuthung, die sich auf Ridolf's Zeugniss stïtzen kann, stellten sie folgende historische Scenen dar: Kaiser Sigismund macht Giovanni Francesco Gonzaga zum Marchese; Lodovico Gonzaga erzwingt den Uebergang über die Etsch gegen die Venezianer; Federico Gonzaga vertreibt die Schweizer von Legnano; der Sieg bei Taro. Guglielmo hatte diese Darstellungen bei einem Besuche in Venedig persönlich bestellt.

1579. October und November. Verhandlungen von Mantua aus über vier weitere, die Gouzaga's verherrlichenden Gemälde. (Gaye, Carteggio III, 428. 433. D'Areo: delle arti e degli artifici di Mantova II, 141. Luzio: ebenda.)

1580. Mai sind die Gemälde fertig. Sie stellten Thaten des Federigo und Francesco Gonzaga dar: die Belagerung von Parma (1521), die 
Einnahme von Mailand (1521), die Vertheidigung Pavia's gegen die Franzosen (1522) und einen Sieg Francesco's. (Luzio: ebenda.)

1580. September ging Tintoretto selbst mit seiner Familie nach Mantua, wo er die Gemälde aufhing, Portraite des Guglielmo und anderer Gonzaga's anfertigte und von dem Herzog bei der Anlage von Bauten zu Rathe gezogen wurde. (Lazio ebenda, Ridolfi II, 26.)

1580. Portraits des Vincenzo Morosini im Dogenpalast. Mit der Jahreszahl bezeichnet.

1580. Die $\mathrm{h}$. Giustina mit den Tesorieri in der Aksdemie zu Venedig. Datirt.

Vor 1581, weil von Sansovino in seiner Venezia erwähnt, sind folgende Bilder entstanden (es können darunter natürlich auch frühe Werke des Meisters sein, da Vasari 1568 ja nur einige wenige Werke angeführt hat):

Madonna mit Heiligen in San Benedetto.

Geburt Christi in San Benedetto.

Himmelfahrt der Maria in den Crociferi. 1657 in die Gesuati überführt.

Beschneidung Christi in den Crociferi. 1657 in die Gesuati überführt.

Disputation der h. Katharina in S. Daniello.

H. Rochus mit Heiligen in S. Felice.

H. Demetrius mit Stifter in S. Felice (noch dort vorhanden).

H. Katharina vor dem Rad knieend. In S. Geminisno.

Christus am Kreuz in den Gesuati (noch dort vorhanden).

Taufe Christi. In der Taufkapelle von S. Giacomo dall'Orio (noch dort vorhanden).

Dreieinigkeit mit Heiligen in S. Girolamo.

Ausschiffung der h. Ursula in den Incurabili (noch dort vorhanden).

H. Michael in S. Leone.

Bekehrung der Magdalena. In S. Maria Maddalena.

Tod der Magdalena. Ebendaselbat.

Abendmabl. In S. Margherita.

Anbetung. In S. Margherita.

Fusswaschung. In S. Margherita.

Orgelflügel mit vier Evangelisten (noch vorhanden) und Bekehrung Saul's in S. Maria Zobenigo.

H. Marziale mit Heiligen in S. Marziliano (noch dort vorhanden).

Bild (Fusswaschung?) in S. Moisé (noch dort vorhanden).

Kreuzigung in $\mathrm{S}$. Severo.

Himmelfahrt der Maria in S. Stefano (8. Stin).

Geburt Johl̀nis des Täafers in S. Zacearia. (Noch dort.)

Deckenbilder in der Scuola di San Fantino.

Lanette in den Procurazien.

Portraits der Dogen, die zuvor Procuratoren waren. In den Procurazien. 
In der Seuola di S. Roceo erwähnt Sansovino uur die Gemälde im Sitzungazimmer: die Decke, die Kreazigung, Ecee homo, Christas vor Pilatus and das Gebet in Gethsemane. Bezüglich des letzteren begeht Sansovino einen Irrthum: er meint die Kreuztragung. Da keines der grossen Wandgemälde im oberen und unteren Saale von Sansovino genannt wird und er dieselben schwerlich versohwiegen haben würde, so flüchtig. auch er sonst bei der Frwähnung von Kunstwerken ist, so dirfte also 1581 noch keines derselben in der Scuola di $S$. Roceo aufgehängt gewesen sein. - Auch von den Gemälden in den 1578 und 1579 wieder hergestellten Sale del Gran Consiglio und dello Scrutinio wird noch keines erwähnt.

1580-1594 (?) führt Jacopo die Wandbilder in dem oberen und unteren Saale der Scuola di S. Roceo aus. Fast sus allen Jahren bis 1 ä93 sind Zahlungsanweisungen in den Aeten der Brïderschaft enthalten. Zumeist sind es die Zahlangen der jährlichen Provision von 100 Ducaten, daneben aber auch solche für die besonders kostbare Farbe des Ultramarinblau. Wann die letzten Gemälde vollendet wurden, ist vorläufig nicht zu sagen.

Zwischen 1580 und 1585 dürften die Deckenbilder der Sala del Gran Consiglio entstanden sein, deren grösstes die Apotheose Venedig's mit dem Dogen Niccold da Ponte, der von 1578-1585 regierte, zeigt. Die anderen historischen Gemälde in dem Saale und in der Sala dello Scrutinio, welebe Bardi 1587 erwähnt, die damals aber vermuthlich nur geplant, nicht ausgeführt waren - woraus sich Bardi's falsche Angaben erklären würden sind nicht von Tintoretto.

Zwisehen 1578 und 1585 entstand das Portrait des Dogen Niccolo da Ponte (in mehreren Exemplaren erhalten).

Zwịchen 1578 und 1585 vermathlich auch die grossen Repräsentationsbilder in der Sala del Collegio des Dogenpalastes, soweit hier überhaupt Tintoretto's Hand oder Geist zu finden ist: Andrea Griti vor der Madonna, Francesco Donato bei der Verlobung der h. Katharina, Alvise Mocenigo vor der Vision Christi und Niccolo da Ponte's Verherrliehung. Man darf wohl annehmen, dass letzterer diese Gemälde hat ausführen lassen.

1580. Portrait des Vincenzo Morosini im Dogenpalast. Mit der Jahreszahl bezeichnet.

1585. Portrait in der Galerie zu Cassel. N. 497. Ist es Tintoretto?

1585. Die Portraits der japanischen Gesandten, welche damals Venedig besuchten. (Ridolfi. Cicogna, Iscrizioni V, 649).

Zwischen 1585 und 1594. Portrait des Dogen Pasquale Cicogna.

Zwischen 1583 und 1588. Die Erscheinung des Auferstehenden vor Vincenzo Morosini und seiner Familie. In S. Giorgio. Die betreffende Kapelle des h. Andreas wurde 1583 dem Vincenzo überlassen unter der Bedingung, dass er sie innerhalb dreier Jahre ausschmücke. Vincenzo + 1588. (Cicogna: Iscrizioni IV, 268, 350.)1 
1587, nach. 1587 wurde die Decke im Senatssagle des Dogenpalastes vollendet (Lorenzi S. 504, 505). Man darf wohl annehmen, dass damals etwa auch die Deckenbilder gemalt wurden, deren mittelstes: Venedig als Herrscherin der Meere, wenigstens der Zeichnung nach, von Jacopo entworfen wurde. - Die anderen im Senatssaale ihm zugesehriebenen Gemälde: Pietro Loredano vor dem hersbschwebenden Mareus und die Pietà mit dem Dogen Pietro Lando und Mare Ántonio Trevisano rühren nicht von ihm her.

1587. In einem Briefe des Gesandten Hieronimo Lippomanno an seinen Bruder heisst es: Tintoretto arbeite an einer Skizze des Jüngsten Gerichtes für Philipp II. Velazquez hat sie später gekauft. Es ist die heute in Madrid befindliche Skizze zum Jüngsten Gericht in der Sala del Gran Consiglio (Paul Lefort: Ie musée du Prado. Gazette des beaux-arts. III pér. VIII, S. $472 \mathrm{ff}$.). Diese Nachricht stimmt nicht mit der von Ridolfi (I, S. 330 u. 398; II, 51) überlieferten und heute noch geltenden Tradition, die auf Bardi zurückzuführen ist: Paolo Veronese und Francesco Bassano hätten zuerst den Auftrag auf das „Jüngste Gericht“ erhalten (so bei Bardi: Dichiarazione 1587, S. 46), dann nach dem Tode Paolo's (19. April 1588) habe Tintoretto den Auftrag erhalten. Ridolfi weiss sogar zu erzählen, Prolo habe die Trinität und die Engel malen sollen. Im Leben Bassano's sagt er: es sei nicht zu dieser Mitarbeiterschaft gekommen wegen der Verschiedenheit des Stiles der beiden Künstler. Letzteres erscheint sehr glanbwürdig. Dann hätten wir uns am ersten den Vorgang so zu denken: wie Bardi erzählt, wären zunächst Veronese und Francesco Bassano ausersehen worden für die Arbeit, man hätte diesen Gedanken aber bald aufgegeben und schon 1587 Tintoretto mit dem Gemälde beauftragt, der damals die von Lippomanno gesehene Skizze anfertigte.

1587 und folgende Jahre. Das Paradies im Palazzo ducale.

1588. 1. Mai Zahlung für Ultramarinblau für die Altartafel: Apotheose des h. Rochus in der Seuola di S. Roceo.

1588. Zeichnungen für Mosaiken in $\mathrm{S}$. Marco, und zwar wiederum für das zchon 1577 begonnene Paradies über der Vorhalle (von Bart. Bozza ausgeführt), für die Figuren des Hiob und Jeremias (im Längsschiff von Giannantonio Marini ausgefïhrt), für den Hosea (von Lorenzo Ceceato ausgeführt), für die Darstellungen der Verkündigung, Anbetung der Könige, Verklärung. Darstellung im Tempel und Taufe (von Giannantonio Marini), einige Daratellungen aus der Geschichte der Susanna (schon 1587), der h. Johannes Damascenus (links in der Galerie, von Febo Bozza ausgeführt), und für ein "Schweisstuch mit Engeln" (von Giannantonio Marini ausgefuihrt). (Saccardo: les mosaïques S. 65, 296; 68, 306; 69 $295 ; 68,71,295 ; 82,305$.)

1590. Portrait des Paolo Paruta im Dogenpalast. Mit der Jahreszahl bezeichnet.

1590 stirbt Marietta, Tochter Jacopo's.

1592. Zeichnungen für Mosaiken in S. Marco: Symbolische Figur 
der Kirehe im rechten Seitensehiff (von Giannantonio Marini ausgefihrt) und h. Petrus im Chor (von Arminio Zuceato ausgefuhrt). (Saccardo: les mossíques $\mathrm{S} .68,307,69$.

1592-1594? Wohl die beiden Bilder: Geisselung Christi und Christi Himmelfahrt in der Kirche Redentore. Dieselbe wurde 1592 geweiht.

1593. Das Martyrium des h. Stephanus in S. Giorgio. Der Altar des Stephanus wurde am 1. März 1593 gestiftet. (Cicogna IV, 352.)

1593. 1594. Das Martyrium der h. Cosmas und Damianus in $S$. Giorgio. Der betreffende Altar wurde in den Jahren 1592 oder 1593 errichtet. (Cicogna IV, 353.)

1598. 1594. Die Krönung der Maria mit den h. Benedictinern in S. Giorgio. Der Altar des h. Benedict wurde am 23. Februar 1593 gefertigt. Die Bezahlung an Tintoretto ist im Jahre 1594 mit 150 Ducaten notirt. (Ebendaselbst.)

1594. Die Mannahlese und das Abendmahl in S. Giorgio.

Hier findet sich bei Cicogna eine falsehe alte Angabe aus Olmo's Manuscript: 1564, für 180 Ducaten. Offenbar handelt es sich um einen Irrthum. Es muss 1594 heissen, denn der Chor wird erst in den Jahren 1591 (Bronzen des Girolamo Campagna) und in den folgenden (1594, 1. April die Chorschranken) ausgeschmückt. (Cicogna IV, 265, 344). Damals müssen auch Tintoretto's Gemälde entstanden sein.

1594. Die Zurïckweisung des Opfers Joachim's und die Anbetung der h. drei Könige. Von Tintoretto unvollendet hinterlassen. Eingt in S. Maria maggiore, jetzt in S. Trovaso (Ridolf II, 64. C. Piucco: Archivio storico dell' Arte II serie III, 48).

1594. 30. März. Testament Jacopo's (publicirt bei Zabeo, Elogio und von M. de Mas-Latrie in der Gazette des beaux-arts 1865. XIX, S. 96).

1594. 31. Mai stirbt Tintoretto. (Nach Acten von S. Marzilisno, (vgl. Zabeo), und in den Frari: Proveditori alla Sanita Necrologio 31, 1593-94, 1825, bei Galanti S. 77).

Fast ein halbes Jahrhundert nach dem Tode des Meisters ward dessen Biographie vom Cavaliere Carlo Ridolfi verfasst, und zwar erschien sie zuerst einzeln 1642 , dann 1648 in die Maraviglie dell' arte aufgenommen. Sie darf als eine wenn auch späte, so doch als die wichtiggte Quelle für unsere Kenntniss der Werke Tintoretto's betrachtet werden. Eine leidenschaftliche Bewunderung für den grossen Künstler, der allgemein damals von den venezianischen Malern als der höohste Stolz ihrer Schule betrachtet wurde und dessen Ruhm sich in alle Lande, besonders 
auch durch Velazquez (1629 in Venedig) nach Spanien verbreitet hatte, bewog Ridolfi, eine mögliohst vollständige Aufzählung der Gemälde Jacopo's zu geben. Wir dürfen seinen Angaben vollen Glauben sehenken, da er sich an eine ganz sichere Tradition halten und zudem der Mittheilungen des ihm befreundeten Domenico Tintoretto $(\dagger 1637)$ sich bedienen konnte. Den vereinzelten Angaben der älteren Sehriftsteller stellt er seine höchst umfangreiche Liste der von ihm in Kirchen, öffentlichen Gebüuden und auch privaten Sammlungen aufgesuchten und aller ihm bekannt gewordenen, aus Venedig hinaus gelangten Bilder gegenüber, er bestimmt eine Gruppe von Arbeiten der Jugendzeit und berichtet das freilich nicht Viele, was ihm von charakteristisehen Zügen des Wesens und der Arbeitsweise Jacopo's bekannt geworden war. Leider verzeichnet er aber so gut wie keine Daten.

Den überschwänglichen poetischen Schilderungen der Thätigkeit Tintoretto's, die sich in Marco Boschini's Carta del navegar pitoresco (1660 erschienen) verstreut an vielen Stellen finden, können wir nur verschwindend wenige neue positive Angaben entnehmen, und diese beschränken sich, abgesehen von den mitgetheilten, Jacopo verherrlichenden Urtheilen nambafter Künstler, fast ganz auf graphische Reproductionen Tintoretto'scher Genälde. Aber in seinen Ricche Minere della Pittura Veneziana, die zuerst 1664, dann in zweiter Auflage 1674 erschienen, bringt derselbe Verfasser, der es sich in der Carta del navegar zur Aufgabe gemacht hatte, Vasari's bedingte Auffassung der Venezianischen Malerei mit allen Mitteln der Polemik und des Enthnsiasmus zu widerlegen und entkräften, eine gewissenhafte, trockene Aufzählung aller in Venedig befindlichen Gemälde, und verzeichnet bei dieser Gelegenheit eine Anzahl von Bildern, welche von Ridolfi nicht genannt worden waren. Es empfiehlt sich, diegelben hier anzufuihren:

Portrait Benedict XI. (1303-1304). Im Kloster (Scuola vor dem Capitolo von S. Niccolo) von S. Giovanni e Paolo.

Verkündigung. Im Capitolo di S. Niccolò von S. Giovanni e Paolo.

Mosaiken in S. Marco: Paradies über Vorhalle, die fünf Darstellungen an der Eingangswölbung des Chores, drei Geschichten der Susanna.

Tempelgang der Maria. S. Maria Fava.

Geschichte aus altem Testament. S. Maria Fava.

Himmelfahrt der Maria. Auf Hochaltar von s. Maria Formosa.

Fahne (Gonfalone) mit h. Michael, an den Ecken vier Evangelisten, unten einige Portraits. Beschädigt in der Madonna dell'Orto.

Altargemälde des h. Martin in S. Martino (Isola). „War von Tintoretto, wurde aber von Palma restaurirt." Der Arme, der Mantel empfängt, und ein h. Bischof seien von Jacopo.

di Castello.

Mosaik: Allerheilige, ausgeführt von Arminio Zuccati in \$. Pietro

Christus sitzend mit knieenden Aposteln, und dem heiligen Hiero- 
nymus, Franz, Ludwig und Antonius von Padua. In der Chiesetta vecchis bei Redentore.

Gebet in Gethsemane in S. Silvestro.

Abendmahl. In S. Simeone grande.

Marcus in Luft mit drei Stiftern und zwei Secretären. In den Camerlinghi.

Marcus thronend mit Senatoren. „Pensate la fin." Ebendaselbst (jetzt Berlin).

H. Giustina und Camerlinghi. Ebendaselbst (jetzt Akademie).

Die Heiligen Ludwig, Hieronymus und Andreas im Magistrato dei Governatori delle Entrate (jetzt unter Bonifazio's Namen in der Wiener Akademie).

Auferstehender Christus mit drei Giudici im Magistrato dei Pro veditori sopra le ragioni delle Camere (jetzt Akademie).

Madonna und drei Senatoren. Ebendaselbst (jetzt Akademie).

Anbetung der h. drei Könige (Portraits) im Magistrato sopra i Conti (jetzt Akademie).

Zwei Bilder mit je drei Portraits. Magistrato delle Cazude.

Die Heiligen Mareus und Laurentius als Portraits. Im Magistrato del Monte di sussidio.

Zahlreiche, einzeln namhaft gemachte Procuratorenbildnisse in den Procurazien.

Verkündigung. Kleines Bildchen in der Scuola di S. Francesco.

Zwei Wunder des h. Hieronymus, Ebendaselbst.

Volto santo von Engeln angebetet und Madonna über Thür des Hofes der Scuola dei Lucchesi bei den Servi.

Taberuakel, Riva del vino, Ecke der Calle dei cinque: Verkündigung (aussen), Madonna (innen), an den Flügeln: vier Kirchenväter

Reste von Wandmalerei: h. Christoph (schon von Ridolfi erwähnt) und Schlacht, auf der Maner der Gesuiti, gegenüber Palazzo Zeno.

S. Giosafat und zwei Engel. Im Klostergang von S. Andrea della Certosa.

Portraits von Tintoretto's Frau und Schwiegermutter. Bei Conte Bencio (Carta del navegar 537).

Putten auf Camino, ein Alter voin Tod geknechtet, Wandmalereien an der Casa Lipamana bei S. Fosea.

Beweinung Christi in der Umiltà.

Anbetung der drei Könige in der Chiesa della Desmesse.

Darstellung im Tempel. Ebendaselbst.

Die Ehebrecherin vor Christus. Ebendaselbst.

Zwei der hier neu genannten Bilder sind auch von Martinioni in seinen Anmerkungen zu der von ihm 1663 neu herausgegebenen Venezia des Sansovino verzeichnet: das Abendmahl in S. Simeone grande und die Erscheinung der Madonna vor dem h. Hieronymus in S. Fantino. Im Uebrigen bringt Martinioni keine Ergänzungen zu Ridolf. 
Zu diesen Angaben venezianischer Schriftsteller gesellen sich im XVII. Jahrhundert mancherlei andere, welche nach auswärts gelangte Werke des Meisters betreffen und in Inventaren, Katalogen und Briefen zu finden sind. Dieselben schon hier einzeln anzuführen, wärde zweoklos sein. Nur die wesentliche Grundlage für die Untersuchungen über die einzelnen Schöpfungen sowohl, als auch über die Entwieklung der Tintoretto'schen Kunst sollte hier gegeben werden. Dieser Aufgabe näher zu. treten, sind wir nun berechtigt, und wenden unsere Betrachtung zunächst den in Venedig noch heute befindlichen Gemälden zu.

(Fortsetzung folgt.) 